\section{DIRAC'S REFINED UNIFICATION OF QUANTUM MECHANICS AND SPECIAL RELATIVITY: AN INTERTHEORETIC CONTEXT}

\begin{abstract}
One of the key episodes of history of modern physics - Paul Dirac's startling contrivance of the relativistic theory of the electron - is elicited in the context of lucid epistemological model of mature theory change. The peculiar character of Dirac's synthesis of special relativity and quantum mechanics is revealed by comparison with Einstein's sophisticated methodology of the General Relativity contrivance. The subtle structure of Dirac's scientific research program and first and foremost the odd principles that put up its powerful heuristics is scrutinized with special emphasis on the highly controversial tenet of "mathematical beauty." It is contended that despite the relentless Dirac's remarks denigrating the controversial role of philosophy one can trace its indirect influence through Arthur Eddington's and Hermann Weyl's whimsical mathematical models. Accordingly, the milestones of Dirac's research programme realization in the distinctive context of the applied epistemological doctrine are indicated.
\end{abstract}

Keywords: quantum mechanics; special relativity; synthesis; Dirac; Eddington; Einstein; Weyl

\section{Diracovo vylepšené sjednocení kvantové mechaniky a speciální relativity: interteoretický kontext}

Abstrakt: Jedna $z$ klíčových epizod dèjin moderní fyziky - překvapivý vznik relativistické teorie elektronu Paula Diraca - je analyzována $z$ hlediska epistemologického modelu zmèny zralé teorie. Článek poukazuje na zvláštní charakter Diracova sjednocení speciální relativity a kvantové mechaniky, který se objevuje prri srovnání s Einsteinovou sofistikovanou metodologií v pozadí teorie všseobecné relativity. Rafinovaná struktura Diracova védeckého výzkumného programu a neobvyklé principy, které vybudovaly jeho silnou heuristiku, jsou prozkoumány s di̊razem na vysoce kontroverzní princip „matematické krásy“. V článku tvrdím, že navzdory neutuchajícím Diracovým poznámkám popírajícím roli filosofie lze vysledovat její neprímý vliv prostřednictvím hravých matematických modeli Arthura Eddingtona a Hermanna Weyla. $V$ souladu s tím jsou predstaveny hlavni body Diracova výzkumného programu $z$ hlediska aplikované epistemologické doktriny.

Klíčová slova: kvantová mechanika; speciální relativita; syntéza; Dirac; Eddington; Einstein; Weyl

Republic of Tatarstan

Russian Federation email / nugayevrinat@gmail.com (iD) 0000-0001-9925-2264

\author{
RINAT M. NUGAYEV \\ Volga Region State University \\ Universiade Village 35 \\ 420138 Kazan
}

This work is licenced under the Creative Commons Attribution 4.0 International License. 


\section{Introduction}

Paul Dirac's refined unification of the special theory of relativity (STR) and nonrelativistic quantum mechanics (QM) occupies a peculiar place in the history \& philosophy of science due to the following circumstances.

First, Dirac's skeptical attitude to epistemology is too well-known to dwell on. For instance, the author of this paper cannot indicate either the name of the philosopher, or the name of the philosophical school, to which Dirac referred at least once, not to mention a single humble quotation from any philosophical writing.

The electrical engineer, a guileless graduate of the University of Bristol, was characterized by a markedly caustic attitude towards epistemology, which at best only dealt with "what was, and that, alas, passed without a trace" and could not set the definite and distinctive guidelines necessary for successful research activities. In this respect, Dirac's views apparently were at odds with the Weltanschauung of another smart youngster and one of his strongest competitors - Werner Heisenberg, the future author of Physik und Philosophie, ${ }^{1}$ who often quoted Kant, or, say, Niels Bohr himself, who even mentioned Sören Kierkegaard (not to slight Erwin Schrödinger, who craved for teaching philosophy professionally).

To this one should add the almost complete absence of notebooks, diary entries, etc., which would make it possible to elicit with confidence Dirac's alluring logic of discovery. On the other hand, there is also a direct Dirac's indication that the "true history" of his research does not deviate too much from that which can be reconstructed based on an analysis of his articles. ${ }^{2}$

All of the above not only does not eliminate, but on the contrary - conditions - the need for a rational reconstruction of the Dirac synthesis of SRT and nonrelativistic QM, especially for comparing it with classical samples of synthesis and unfolding its specificity. It is also fascinating due to the significant asset that Dirac's thought-provoking relativistic theory of the electron played in the second scientific revolution in general and in the contrivance of both QED (quantum electrodynamics) and QFT (quantum field theory).

Hence, the aim of this paper is to try, despite the obvious absence of direct instructions from Dirac, to highlight the basic heuristic principles of the synthesis of SRT and nonrelativistic QM (first section) and lay out how they were implemented in actual scientific practice (second section).

\footnotetext{
${ }^{1}$ Werner Heisenberg, Physik und Philosophie (Stuttgart: Hirzel, 2000).

${ }^{2}$ Helge Kragh, "The Genesis of Dirac's Relativistic Theory of Electrons," Archive for History of Exact Sciences 24 (1981): 31-52.
} 


\section{Eliciting the Pivotal Heuristic Principles of Paul Dirac's Scientific Research Program}

In my view, one can still admit the existence of the "positive heuristic" of Dirac's SRP (scientific research program), which, without any significant changes, permeated all of his creative work.

(I) As the first principle, one can single out Dirac's blunt and solid views about the gradual, evolutionary development of science and the need for gradual (piecemeal) changes in the content of the scientific theories. ${ }^{3}$ In this respect, Paul Dirac's unobtrusive views were definitely at odds with those of his friend, colleague and opponent, Werner Heisenberg. Heisenberg, with his staggering enthusiasm, advocated a holistic interpretation of physical theory. The latter consisted, first of all, in a typical Duhemian thesis on the integrity of the content of a scientific theory, when the slightest changes in its content in a discreet place instantly led to corresponding changes in all the nooks. Accordingly, a scientific revolution incarnates a simultaneous (and abrupt) change of the entire "paradigm" at once. Incidentally, one of the important consequences of this circumstance is the renowned "KuhnFeyerabend thesis" on the incommensurability of the contents of the "old" and "new" mellow paradigms.

Dirac, starting from his early "postgraduate" articles, always constantly - and until the end of his life - stressed the continuity of QM and classical mechanics. ${ }^{4}$

Ultimately, in more strict and complete terms, in his staggering single paper devoted as a matter of fact to philosophy of physics and encouragingly titled "The Relation between Mathematics and Physics" Dirac maintains:

The difference [between classical and quantum mechanics] may be expressed concisely, but in a rather abstract way, by saying that dynamical variables in quantum mechanics are subject to an algebra in which the commutative axiom of multiplication does not hold. Apart from this, there is an extremely close formal analogy between quantum mechanics and the old mechanics. In fact, it is remarkable how adaptable the old mechanics is to the generalization of non-

\footnotetext{
${ }^{3}$ Alisa Bokulich, "Open or Closed? Dirac, Heisenberg and the Relation between Classical and Quantum Mechanics," Studies in History and Philosophy of Science 35, no. 3 (2004): 377-96.

${ }^{4}$ Paul Dirac, "The Physical Interpretation of the Quantum Dynamics," Proceedings of the Royal Society of London, A 113 (1927): 621.
} 
commutative algebra. All the elegant features of the old mechanics can be carried over to the new mechanics, where they reappear with an enhanced beauty. ${ }^{5}$

(II) The second in a row (but not in importance!) - put forward by Dirac as a pivot - the so-called principle of mathematical beauty. This principle is so dear to the QED father that: "A theory with mathematical beauty is more likely to be correct that an ugly one that fits some experimental data." ${ }^{6}$

Recently, Olivier Darrigol, the renowned French historian and philosopher of science, discovered an unpublished version of Dirac's article, which contained the following distinctive phrase, shedding some additional light on Dirac's comprehension of this most important heuristic principle for him:

The quantum theory has now reached a form, in which it is as beautiful, and in certain respects more beautiful than the classical theory. This has been brought about by the fact that the new quantum theory requires very few changes from the classical theory, these changes being of a fundamental nature, so that many features of the classical theory to which it owes its attractiveness can be taken over unchanged into the quantum theory. ${ }^{7}$

(III) The third, all appearance, no less significant principle of the Dirac heuristic is closely related to those considered above: the general theory of relativity (GTR) constitutes a sublime ideal, an unsurpassed specimen of mathematical beauty. Accordingly, Einstein's experience of constructing it was of decisive importance for the problem of relativistic electron contrivance and hence for proper combining SRT and nonrelativistic QM. Especially strongly, according to his tentative biographers, Dirac was staggered by the dazzling fact that the GTR was created without any visible connection with experiment, and, as Arthur Eddington never tired of emphasizing, by no means as a direct generalization of experimental data. This, nevertheless, did not prevent the GTR not only to perfectly explain the anomalous precession of the perihelion of Mercury, known for more than half a century, but also to brilliantly predict the unrehearsed deflection of light rays in the gravitational field of the Sun. The bold prediction was famously confirmed in 1919 by an expedition of British astronomers led by Sir Arthur Eddington.

\footnotetext{
${ }^{5}$ Paul Dirac, "The Relation between Mathematics and Physics," Proceedings of the Royal Society of Edinburgh, A 59 (1938-1939): 125 (my emphasis).

${ }^{6}$ Paul Dirac (1970) quoted in Bokulich, “Open or Closed?," 377-96.

${ }^{7}$ Paul Direc (1927) quoted in Olivier Darrigol, From c-Numbers to q-Numbers: The Classical Analogy in the History of Quantum Theory (Berkeley: University of California Press, 1992), 435 .
} 
Extremely important for the professional formation of the English theoretical physicist was the fact that from the very beginning of his acquaintance with the GTR, Dirac was so fascinated by it (not without the influence of the books, lectures and discussions with Arthur Eddington) that he dreamed of studying this very theory in Cambridge. And only when it became clear that the corresponding tutor was absent there, the former unemployed electrical engineer was forced to accept Ralph Fowler's magnanimous offer to dwell into the bizarre realm of quantum theory. But even in the process of preparing his $\mathrm{PhD}$, Dirac could not deny himself the pleasure of attending the fascinating lectures on GTR, delivered by her greatest English expert in the field, prof. Arthur Eddington. Moreover, he had the lucky opportunity to discuss its foundations in personal meetings. But no less important, from my point of view, is the fact that Dirac got acquainted with GTR not, so to speak, "in the original," but only through the prism of the distinctive writings of A. Eddington, who, as a specialist in the GTR subtle field, was known in the post-war England "even more than Einstein himself."

We really had no chance to understand relativity properly until 1923, when Eddington published his book The Mathematical Theory of Relativity which contained all the information needed for a proper understanding of the basis of the theory. This mathematical information was interspersed with a lot of philosophy. Eddington had his own philosophical views, which, I believe, were somewhat different from Einstein's, but developed from them. ${ }^{8}$

An intriguing quote. What kind of queer Eddington's "philosophy of relativity" the eccentric creator of the theory of the relativistic electron had in mind? - In my view, another important (and single!) quote from Dirac should come to the rescue. Indeed, back in 1931, Dirac prefaced his famous magnetic monopole article with the following presentiments, which anticipated the future advancement of theoretical physics. He had stressed that the upcoming changes in the style of scientific research promised to be so radical that they may be outside the shabby boundaries of human intelligence. And the latter will be able to grasp the content of these ideas only with the help of the rickety crutches of mathematical speculation. In light of the upcoming shocks, the practical Dirac discreetly proposed a roundabout way, in which "the most effective method of moving forward" should be

\footnotetext{
${ }^{8}$ Paul Dirac quoted in Thomas Ryckman, The Reign of Relativity. Philosophy in Physics 19151925 (Oxford: Oxford University Press, 1982), 182.
} 
to perfect advance the mathematical formalism that forms the existing basis of theoretical physics, and after each success in this direction, to try to interpret the new mathematical features in terms of physical entities (by a process like Eddington's Principle of Identification). ${ }^{9}$

So, what is this alluring Principle? - Eddington himself, explaining it, referred in his epoch-making monograph primarily to the mathematical identification of the vector and tensor structures of his abstruse "infinitesimal world geometry" with the subtle structures of gravity and electromagnetism (i.e., with the structures of unified theories) pioneered by Hermann Weyl. ${ }^{10}$ Despite the deplorable fact that the notorious Weyl's "unified field theory" turned out to be unrealizable, this did not obscure the attractiveness of the source of Eddington's and Weyl's inspiration - the alluring history of the GTR genesis.

Indeed, Einstein's so-called "geometrization" of the gravitational field in 1914-1915 (the 1913 contrivance of the notorious "Entwurf" first together with Marcel Grossman, and then the 1915 GTR) had provided a model (a "paradigm") of all the entire geometric unification of modern physics. Note that the way of using abstract mathematics to make progress in physics is far older than from Einstein's works and dates back to Newton from $17^{\text {th }}$ century who had to contrive radically new abstract mathematics (infinitesimal calculus) to describe his theories. But in relation to the case in question let us recall ${ }^{11}$ that the deft construction of the GTR fundamental theoretical scheme was due to the successive synthesis of the "non-metric" partial theoretical schemes of Abraham, Nordström, and Einstein himself, advanced in 1907-1914. The staggering synthesis, which culminated in finding out the stupendous Einstein-Hilbert equations, turned out to be possible only by constructing a basic "hybrid" or, in A. Eddington's terms, a "dual" theoretical abstract object - the metric tensor $g_{\mu v}$. It is the latter that deftly combines "geometry" and "physics." On the one hand, this tensor is a purely geometric quantity that masterly characterizes the measured lengths, distances, and times. But, on the other hand, $g_{\mu \nu}$ are ten independent quantities representing the "potentials of the gravitational field," the values of which at each point in space-time are determined by the nature of the distribution of

\footnotetext{
9 Paul Dirac, "Quantized Singularities in the Electromagnetic Field," Proceedings of Royal Society of London, A 133 (1931): 60 (my emphasis).

${ }^{10}$ Hermann Weyl, Raum, Zeit, Materie (Berlin: Springer, 1919).

${ }^{11}$ Rinat M. Nugayev, Einstein's Revolution: A Study in Theory Unification (Sharjah: Bentham Science Publishers, 2018).
} 
masses and energies, represented by the energy-momentum tensor $T_{\mu \nu}$. In this case, the strength of the gravitational field is described by the curvature tensor $R_{\mu v}$, symmetrically containing the derivatives with respect to all the 4 coordinates of the metric tensor.

As a result, the hybrid character of the metric tensor as a terse compendium of field quantities is expressed in the fact that it was "geometrized" in the GTR, i.e., embedded in the space-time geometry. At the same time, Eddington singled out the special asset of differential geometry in the Weyl-Husserl construction (world building) of the "physical world": those theoretical objects are "a synthesis of all aspects" in relation to all kinds of observers. In the popular science monograph Space, Time and Gravity, which was published in Cambridge in 1920 and anticipated The Mathematical Theory of Relativity (1923), Eddington stressed:

Physical reality is the synthesis of all physical aspects of Nature [...]. There may be some illusion in these experimental deductions [i.e., the GTR queer consequences like Mercury perihelion precession or the light bending in the gravitational field of the Sun]; but if not, it must be admitted that the physical reality corresponding to light must be some synthesis comprehending both these appearances [...]. Reality is only obtained when all conceivable points of view have been combined. ${ }^{12}$

Consequently, "Ultimately, if this 'synthesis' is done correctly, physical quantities must be represented by tensors in 4-dimensional space-time." ${ }^{13}$ Hence:

the four-dimensional geometric representation of the world of Minkowski is a "synthesis" required for taking into account the measurements of observers in all states of uniform motion with respect to one another. ${ }^{14}$

Let me arrange the above cumulus by highlighting those main stages of the genesis of any successful mature theory that played a decisive role in the creation of Dirac's masterpiece.

(i) The step-by-step contrivance of a throng of "hybrid" theoretical schemes, caused by the inevitable "encounter" of various pivotal research traditions (such as statistical mechanics vs. Maxwellian electrodynamics; or

\footnotetext{
${ }^{12}$ Arthur Eddington, Space, Time and Gravitation. An Outline of General Relativity Theory (Cambridge: Cambridge University Press, 1920), 182 (my emphasis).

${ }^{13}$ Arthur Eddington quoted in Ryckman, Reign of Relativity, 198.

${ }^{14}$ Ibid., 185.
} 
Newtonian theory of gravitation vs. SRT, or SRT vs. nonrelativistic QM). Each of the hybrid schemes possesses the advantages of its own and describes its own subject area, so that there is a stiff necessity for their synthesis and for constructing a global theoretical scheme with the relations between the theoretical objects described by the global theory fundamental equations. In the case of the GTR genesis, these hybrid theoretical schemes were represented by the artful non-metric theories of Abraham, Nordström, and Einstein; in the case of Dirac synthesis the hybrid schemes were incarnated by Klein-Gordon-Fock (1926) and Pauli-Heisenberg-Jordan (1926) halfphenomenological theories.

(ii) It turns out that the direct resolution of the problem situation due to the elevating of the basic objects of any one of the created hybrid theoretical schemes on the pedestal of the global theory simply "does not work." Gradually, the understanding comes that the desired synthesis is possible only due to the "radical transformation" (Dirac), i.e., due to the involvement of some substantially novel branch of mathematics. Its conceptual system is able to generate a "neutral language" for describing, comparing and "reconciling" all the hybrid schemes obtained. For instance, when Maxwell had contrived his celebrated fundamental theoretical scheme ${ }^{15}$ of classical electrodynamics, the language of vector calculus (represented by the equations of hydrodynamics with its operations $\operatorname{div} \boldsymbol{E}$ and $\operatorname{rot} \boldsymbol{H}$ ) functioned as a neutral language ${ }^{16}$. In the history of the GTR genesis, the subtle notions of Riemannian geometry and tensor calculus also functioned as a neutral language; it is tensors that constitute the geometric objects, the relations between which are preserved in the transition from one non-inertial frame of reference to another. In the genesis of Dirac's theory, the role of such a language was played by the language of spinor calculus with its Clifford algebra, which was referred to in the works of W. Pauli in 1926.

(iii) The acquired neutral language is applied to generalize all the available hybrid schemes and find the simplest equation containing the hybrid regularities encountered in the form of their particular cases (Einstein \& Grossman's "Entwurf"; Dirac's relativistic electron equation). Such a generalization takes place, as a rule, in one of the encountered hybrid theoretical schemes, and at first has a local character, but eventually acquires a global significance. The object lesson is Abraham's deft conjecture, which general-

\footnotetext{
${ }^{15}$ Vyacheslav Stepin, Theoretical Knowledge (Netherlands: Springer, 2005).

${ }^{16}$ Rinat M. Nugayev, "Communicative Rationality of the Maxwellian Revolution," Foundations of Science 20, no. 4 (2015): 447-78.
} 
ized the four-dimensional Minkowski metric to the case of the metric tensor dependence on coordinates, or the introduction of the four-dimensional function $\psi=\psi\left(x_{0}, x_{1}, x_{2}, x_{3}\right)$ by Dirac.

(iv) The crucial stage is taking place. Among the mathematical objects, the relations between which are described by the proposed equations of the global theory, it is necessary to find an object that possesses the properties of the basic object of the global theoretical scheme. Specifically, it is necessary to reveal the "dual" (according to Eddington) or "hybrid" (in our terminology) nature of the theoretical object and display that it simultaneously possesses certain "geometric" properties, transforming itself as a tensor $g_{\mu v}$ and, on the other hand, has "physical" properties also, since it is transformed as an irreducible representation of the Poincaré group, the Lorentz group, etc. This elucidation of the so-called "physical," operational sense of mathematical terms should allow comparing the obtained quantitative relationships with experimental data.

Accordingly, in Dirac's theory of the relativistic electron, the wave functions are described by functions $\psi$ (the so-called "bispinors"), the components of which are complex numbers. Moreover, both in Dirac's theory and in QFT in general, bispinors are connected with such fundamental particles as electrons and quarks. Under transformations of the Lorentz group, which describe the symmetries of the Minkowski space, the bispinors are transformed as specific irreducible representations of this group - as the set of operators in the Hilbert space. It is the symmetries of flat space-time that allow, in accordance with Noether's celebrated theorem, electrons and quarks to possess such conserved properties as mass, spin, and charge.

Therefore, $\psi$ - the pivotal mathematical construct of the Dirac equation - has an explicit dual structure. On the one hand, it is a typical bispinor, which, when the coordinate system changes, including rotations in 3-dimensional space and transformations between frames of reference moving rapidly relative to each other, is transformed not as a vector of ordinary space, but as a mathematical object in a special space called "internal," not intersecting with the usual, "external" space. But, on the other hand, the same $\psi$ is, in the first approximation, the amplitude of the probability of one electron, associated with the density of the charge and current of the Dirac particle. By virtue of the conservation of charge, the quantity that is considered to be the total probability of finding a particle is also conserved. Accordingly, Dirac's theory not only featured the geometric properties of bispinors, but was also able to predict the magnetic moment of an electron, 
as well as the fine structure of lines in the spectrum of atoms, and, most importantly, the existence of antiparticles (or positrons).

\section{The Milestones of Dirac's Electron Theory Contrivance}

By 1928 things in the queer field of quantum theory were as follows. The first pioneering attempts by Planck (1900), Einstein (1905), and Bohr (1913), related to the so-called "old quantum theory," 17 gave way to a relatively calm stage of accumulation of abundant experimental data within the framework of the new paradigm. The stage culminated in the contrivance of two perfect and complete nonrelativistic QM versions proffered by Schrödinger and Heisenberg in 1926; and Dirac (1927) had famously laid out that these options are empirically equivalent. ${ }^{18}$

For obvious reasons, the subject was broached on the creation of relativistic QM. Namely, any correct quantum theory should satisfy the requirement of relativity: the laws of motion that take place in one inertial frame of reference must also be fulfilled in all the other inertial ones. Relativistic quantum theory must be formulated in a Lorentz-invariant form. ${ }^{19}$ "This is necessary in order that the theory may apply to high-speed particles." ${ }^{20}$

As a result of the first, direct attempts to combine the principles of SRT and QM, the following hybrid theoretical schemes were constructed. First, Erwin Schrödinger himself, along with deriving his renowned equation in the spring of 1926, attempted to obtain a kind of its relativistic prototype by inserting relativistic corrections to the Hamilton-Jacobi equation for an electron in an electromagnetic field. ${ }^{21}$ Indeed, SRT requires that the energy of a free particle be described by the expression $H^{2}=c^{2} \boldsymbol{p}^{2}+m^{2} c^{2}$. To obtain a QM analogue of this equation, one must replace the corresponding variables with operators representing the derivatives in all 4 coordinates. As a result, we have

\footnotetext{
${ }^{17}$ Rinat M. Nugayev, The Planck-Einstein Breakthrough: Reconciliation of the Pivotal Research Programs of the Classical Physics (Montreal: Minkowski Institute Press, 2020).

${ }^{18}$ Dirac, "Physical Interpretation of the Quantum Dynamics," 621-41.

${ }^{19}$ David Bjorken and Sydney Drell, Relativistic Quantum Mechanics (New York: McGraw-Hill Education, 1998).

${ }^{20}$ Paul Dirac, The Principles of Quantum Mechanics International Series of Monographs on Physics (Oxford: Clarendon Press, 1981), 253.

${ }^{21}$ Erwin Schrödinger, "Quantisierung als Eigenwertproblem," Annalen der Physik 384, no. 4 (1926): 361-76.
} 


$$
\Delta \psi-\left(1 / c^{2}\right) \partial^{2} \psi / \partial t^{2}-m^{4} c^{4} / h^{2}=0
$$

This relation, also derived by a number of other scholars, back in 1926, was christened the "Klein-Gordon-Fock (KGF) equation." In the textbooks, it is taken as an equation describing the motion of a scalar particle, and the failure of the KGF equation is commonly associated with its inability to theoretically reproduce the spin of particles. But that is inaccurate. The KGF equation should be comprehended from the very beginning as the equation of a spinless particle. Its abandonment by Schrödinger was, however, due to the fact that this equation could not describe strictly one-particle system: it did not preserve what non-relativistic theory considered a probability of particle occurrence anywhere in space. Later, however, it turned out that this property is related to the fact that relativistic quantum theory does not preserve the number of particles. It describes the particle as a quantum of fields that can arise and wear off, while maintaining the total energy. Although Dirac's equation at first seemed appropriate to describe a system of a single electron (it preserves probability), today we know that it actually features quantum electron-positron field (similar to KGF equation that describes the field of scalar particles).

Secondly, in the same year, Wolfgang Pauli aspired to improve the spin hypothesis put forward by Uhlenbeck and Goudsmit which qualitatively adequately explained the spectral data related to the so-called "anomalous Zeeman effect." To do this, he aptly modified the Schrödinger equation by introducing into the Hamiltonian the terms containing the so-called "spin operators" described by 2 -dimensional matrices. ${ }^{22}$ In a nutshell, Pauli's methodology can be summarized as follows. Instead of the unperturbed Hamiltonian $H_{0}$, representing the basic state of the electron as a quantum system, the final Hamiltonian $H=H_{0}+H_{1}+H_{2}$ was concocted, where $H_{1}$ exhibits the contribution from the magnetic field and $\mathrm{H}_{2}$ is the contribution characterizing the electron spin. Alas, despite the apparent empirical success, in theoretical respect, this theory, even masterly improved by W. Heisenberg and P. Jordan, ${ }^{23}$ turned out to be unsatisfactory also.

As a result, by 1928, the problem situation was as follows. Two typical hybrid theoretical schemes had been tentatively invented that strived to merge SRT and QM by introducing artless relativistic ad hoc corrections to

\footnotetext{
22 Wolfgang Pauli, "Über das Wasserstoffspektrum vom Standpunkt der neuen Quantenmechanik," Zeitschrift fur Physik 36 (1926): 336-63.

${ }^{23}$ Werner Heisenberg and Pascual Jordan, "Andwendung der Quantenmechanik auf der Problem der anomalen Zeemaneffekte," Zeitschrift fur Physik 37 (1926): 263-77.
} 
certain parts of the Hamiltonian in order to obtain at last the Schrödinger equation describing a relativistic electron. But all these attempts failed. On the one hand, the partial theoretical Klein-Gordon-Fock scheme, perfectly consistent with the principles of STR, diverged not only from experimental data, but - deeper - from the QM principles. On the other hand, the partial theoretical Pauli-Heisenberg-Jordan abstruse scheme, which perfectly explained the splitting of spectral lines and, therefore, was consistent with the QM bizarre principles, diverged, in turn, with the fine principles of STR.

The general reason for the failure was the hybrid, temporary nature of the results obtained, which were worked out through partial modifications of entrenched theoretical traditions. An embracing approach was needed, i.e., the advancement of some general heuristic principle, analogous to Einstein's celebrated "principle of equivalence" (with the identity of gravity and inertia), and its implementation in a sequence of more and more perfect theories. The systematic application of this principle was to gradually lead to the finding of general equations describing the relationship between certain mathematical constructs. Raising these constructs to the rank of abstract objects of the global theoretical scheme, and their subsequent operationalization should have made it possible to derive such consequences that could be compared with experimental data. All these sober requirements were met by Paul Dirac's masterpiece "The Quantum Theory of the Electron," ${ }^{24}$ received by the editorial staff of the Proceedings of the Royal Society of London on January 2, 1928.

Dirac's whimsical theory, the role of the "ontological" GTR "principle of equivalence" was played now by the epistemological "principle of mathematical beauty," which in many respects was reduced, as noted earlier, to the requirement of making minimal, in comparison with classical theories, changes in a new, non-classical theory. But this requirement cannot exhaust, in my view, the content of this heuristic principle, which is most important for Dirac. It is also necessary to take into account its "synthetic," Eddingtonian and Weylean, subtle aspects. The latter consist in the appeal, when formulating one's equations, to such a mathematical language that is capable of realizing a true synthesis of "geometry" and "physics" by introducing mathematical constructs that effectively integrate both mathematical, transformational, and purely physical, "material" properties.

${ }^{24}$ Paul Dirac, "The Quantum Theory of the Electron," Proceedings of the Royal Society of London, A 117 (1928): 610-24. 
Therefore, of undoubted interest is the hypothesis of the prominent "Dirac scholar" - the Danish historian of science Helge Kragh that, while constructing the fundamental theoretical scheme of his theory, Dirac may have turned to the work of German mathematicians of the $19^{\text {th }}$ century related to Clifford algebras. ${ }^{25}$ Another source of mathematical inspiration ${ }^{26}$ could have been for Dirac the ideas of the English mathematician Henry Baker, set forth in his Principles of Geometry, to which Dirac referred back in 1925 during the advancement of his q-number version of QM. In this book, Dirac could discover the $2 \times 2$ matrices (similar to Pauli matrices), and even the $4 \times 4$ matrices similar to those properly used in the 1928 paper.

Be that as it may, the "mathematical beauty" apparently consists not only in obtaining the maximum number of results with minimal means, but also in the ability to embrace ideas that seem to be in no way related to each other with one elegant gesture. Needless to say that the need for synthesis played a special role for Dirac:

I find this position [the paradox in the mechanical worldview caused by the peculiar role of initial conditions] very unsatisfactory philosophically, as it goes against all ideas of the unity of nature. ${ }^{27}$

Examples of such an integral mathematical language are the vector calculus, represented by hydraulic Maxwellian models, as well as the GTR tensor calculus by A. Einstein. It is hard not to recall Dirac, who expressed himself in his single philosophical article "The Relationship between Mathematics and Physics" very clearly and frankly as usual:

Pure mathematics and physics are becoming more closely connected, though their methods remain different. One may describe the situation by saying that the mathematician plays a game in which he himself invents the rules while the physicist plays a game in which the rules are provided by Nature, but as time goes on it becomes increasingly evident that the rules which the mathematician finds interesting are the same as those which Nature has chosen. ${ }^{28}$

Pauli's conspicuous theory could have facilely become the starting material for the construction of the relativistic theory of the electron: it was

\footnotetext{
${ }^{25}$ Helge Kragh, Dirac. A Scientific Biography (New York: Cornell University, 1990).

${ }^{26}$ Jagdish Mehra and Helmut Rechenberg, The Historical Development of Quantum Theory. Volume 6: The Completion of Quantum Mechanics 1926-1941 (New York: Springer, 2000), 294.

${ }^{27}$ Dirac, "Relation between Mathematics and Physics," 127 (my emphasis).

${ }^{28}$ Ibid., 248
} 
merely necessary to formulate it in a Lorentz-invariant way, generalizing the $2 \times 2$ matrices to the $4 \times 4$ case. But Dirac claimed more. The first stage of his research consisted in the introduction of a substantially novel mathematical construct: a 4-component wave function $\psi=\left(\psi_{1}, \psi_{2}, \psi_{3}, \psi_{4}\right)$. This move was extremely bold and risky, since there was no empirical indication in favour of such a generalization. After all, the spin of an electron could only take two values, not four.

The next steps, of course, consisted in both relativistic generalizations of two-dimensional Pauli matrices to 4 dimensions, and in symmetric ranking of all derivatives, which should have looked, in accordance with relativistic invariance, in the same way, etc. (The technical details are proffered by related textbooks like, ${ }^{29}$ or better yet, by meticulous historical-scientific accounts like. ${ }^{30}$ ) As a result, Dirac came to the following relativistic equation for a free electron

$$
\left[p_{0}-\rho_{1}(\boldsymbol{\sigma}, \boldsymbol{p})-\rho_{3} \mathrm{mc}\right] \psi=0,
$$

where $p_{0}=$ ih $\partial / c \partial t, \boldsymbol{p}=\left(p_{1}, p_{2}, p_{3}\right) ; p_{r}=-\mathrm{ih} \frac{\partial}{c \partial x^{r}}, \mathrm{r}=1,2,3 ; \boldsymbol{\sigma}=\left(\sigma_{1}, \sigma_{2}, \sigma_{3}\right)$,

and the exact cumbersome expressions for $\sigma$ and $\rho(4 \times 4)$ matrices can be found in Dirac's stout book ${ }^{31}$ on pages $253-55$. Specifically, $\rho_{3}=\operatorname{diag}\{1,1,-1$, $-1\}, \sigma_{3}=\operatorname{diag}\{1,-1,1,-1\}$, while

$$
\sigma_{1}=\left(\begin{array}{cccc}
0 & 1 & 0 & 0 \\
1 & 0 & 0 & 0 \\
0 & 0 & 0 & 1 \\
0 & 0 & 1 & 0
\end{array}\right) ; \quad \sigma_{2}=\left(\begin{array}{cccc}
0 & -i & 0 & 0 \\
i & 0 & 0 & 0 \\
0 & 0 & - & -i \\
0 & 0 & i & 0
\end{array}\right) ; \quad \rho_{1}=\left(\begin{array}{cccc}
0 & 0 & 1 & 0 \\
0 & 0 & 0 & 1 \\
1 & 0 & 0 & 0 \\
0 & 1 & 0 & 0
\end{array}\right) \text {. }
$$

Note that in modern textbooks Dirac's equation looks like $\left(i \gamma^{\mu} \partial_{\mu}-m\right)$ $\psi=0$, where

$$
\partial_{\mu}=\left(\frac{1}{c} \frac{\partial}{\partial t}, \frac{\partial}{\partial x}, \frac{\partial}{\partial y}, \frac{\partial}{\partial z}\right) ; \quad \gamma^{\mu}=\left(\gamma^{0}, \gamma^{1}, \gamma^{2}, \gamma^{3}\right),
$$

\footnotetext{
${ }^{29}$ Bjorken and Drell, Relativistic Quantum Mechanics.

${ }^{30}$ Kragh, "Genesis of Dirac's Relativistic Theory of Electrons," 31-52. See also Mario Valente, "The Dirac Equation and its Interpretations," preprint, https://www.researchgate.net/ publication/340664785.

${ }^{31}$ Dirac, Principles of Quantum Mechanics, 253-75.
} 


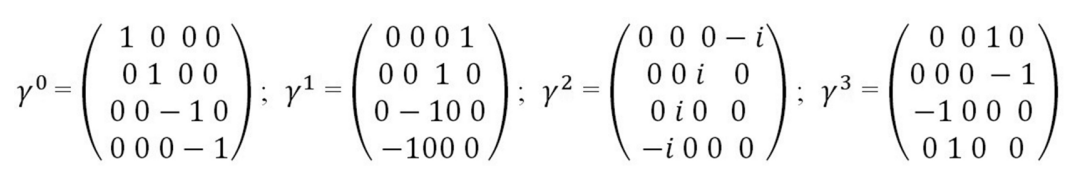

Further, Dirac generalized this equation in the most common way to the case of an electron in an electromagnetic field using the standard replacement of $p_{0}$ with $p_{0}+(e / c) A_{0}$ and $\boldsymbol{p}$ with $\boldsymbol{p}+(e / c) \boldsymbol{A}$. Having squared it, he obtained a significant expression, which contained, in comparison with the Klein-Gordon-Fock and Pauli equations, two completely new terms. Naturally and in a famous way Dirac identified the first term with the electron magnetic moment $(e h / 2 m c) \boldsymbol{\sigma}$. As for the second term, which related to the electric moment, Dirac declared it physically meaningless as containing an imaginary complex number that cannot be part of the real Hamiltonian. This seemingly nondescript detail drops an important hint: Dirac's theory is by no means the result of any irreproachable conclusion either from an experiment, or from a certain deep a priori principle. This is a quirk mathematical surmise, the validity of which still needs to be confirmed by the results of future experiments and calculations. Incidentally, in chapter XI "Relativistic Theory of Electron" of his prominent Principles of Quantum Mechanics, first published in 1930, Dirac had succinctly reproduced the derivation of his pioneer 1927 paper, humbly admitting that its exact form is "just" a guess, albeit well educated and well supported. ${ }^{32}$

In sum, the successful synthesis consisted in getting a miraculous equation, the space-time properties of which were transformed in accordance with the STR, and the quantum properties - in accordance with the QM transformation properties. But the main asset of Dirac's theory consisted, of course, in the theoretical reproduction of the electron spin, which naturally turned out now to be a strictly relativistic consequence of his theory.

I was not interested in bringing the spin of the electron into the wave equation [...]. It was a great surprise for me when I later on discovered that the simplest possible case did involve the spin. ${ }^{33}$

An elegant derivation of the correct spin and magnetic moments of an electron allowed Dirac to easily explain away all the spectroscopic puzzles at hand - the Zeeman and the Paschen-Buck effects, as well as the Sommerfeld

\footnotetext{
${ }^{32}$ Dirac, Principles of Quantum Mechanics, 253-75.

${ }^{33}$ Paul Dirac quoted in Kragh, “Genesis of Dirac's Relativistic Theory of Electrons," 55.
} 
odd formula. But, perhaps, the most unexpected consequence of Dirac's relativistic theory was the staggering prediction of a new type of matter - the so-called "antimatter", the existence of which was not expected by anyone before, but which was confirmed literally several years later. Only after this amazing discovery did all legitimate doubts about the validity of the Dirac synthesis of SRT and QM completely disappear. Associated with this and subsequent achievements is such a characteristic feature of the work of the former electrical engineer as "gradualness" (piecemealness). For instance, to the mathematician Klein, who frivolously tried to unite nonrelativistic QM and GTR in one step at once, Dirac retorted that the main reason for his failure was that he was trying to solve too many problems at once.

One should not try to accomplish too much in one stage. One should separate the difficulties in physics one from another as far as possible, and then dispose of them one by one. ${ }^{34}$

This is exactly what Dirac did. Yet the introduction of spin turned out to be only one of the main benefits of Dirac's equation. The equation naturally ("by itself") explained the spin magnetic moment of an electron that Pauli's equations had inserted only phenomenologically. In this sense, Dirac's equation constituted the first consistent description of particles with spin $\frac{1}{2}$. Though the most amazing implication of Dirac's equation, which is inherently encoded in using 4-component bispinors, is the prediction of antiparticles. Eventually Dirac anticipated them correctly, though initially he had misinterpreted them. From Dirac's whimsical equation to the solid and mature theory of antiparticles further "empirically-progressive problemshifts" were needed. It was necessary to move from a description of quantum systems with a fixed number of particles to "second quantization" of physical fields. Without this crucial step, the relativistic quantum theory was not consistent.

It might be worth mentioning Dirac's major contributions to formulation of the basic mathematical apparatus of quantum theory, which gradually appeared in re-editions of his renowned textbook The Principles of Quantum Mechanics. It should be stressed that Dirac with his distinctive intuitive approach foreshadowed the sort of mathematics that was rigorously contrived only many years later (specifically, the theory of distributions, as well as the theory of so-called "rigged Hilbert space").

${ }^{34}$ Ibid., 40. 


\section{Main Conclusions and Prospects of the Research}

Chapter XI "Relativistic Theory of the Electron" of Dirac's masterpiece The Principles of Quantum Mechanics ${ }^{35}$ first published by the Clarendon Press in 1930, begins as follows:

Let us now try to make the theory invariant under Lorentz transformations, so that it conforms to the special theory of relativity. This is necessary in order that the theory may apply to high-speed particles. ${ }^{36}$

It is no coincidence that this unobtrusive scheme had put up the structure of Dirac's pioneering article "The Quantum Theory of the Electron", published in 1928 in the Proceedings of the Royal Society of London. ${ }^{37}$ The fact serves as a particularly vivid illustration of the need to distinguish between the logic of discovery and the logic of confirmation (the logic of justification) of a scientific hypothesis, which lies at the heart of philosophy of science. It goes without saying that the actual "logic of Dirac's discovery" looked completely different, as it was revealed, for instance, thanks to a plain interview given by Dirac to the historian of science Jagdish Mehra much later.

I was not interested in bringing the spin of the electron into the wave equation, did not consider the question at all and did not make any use of Pauli's work. The reason for this is that my dominating interest was to get a relativistic theory agreeing with my general physical interpretation and transformation theory. I thought that this problem should first be solved in the simplest possible case, which was presumably the spinless particle, and only after that should one go to consider how to bring in spin. ${ }^{38}$

Therefore, the first and most important hallmark of Dirac's stupendous synthesis was that it turned out to be an inextricable continuation of his previous profound studies, which consisted, as is well-known, in successful attempts to reveal the empirical equivalence of the Heisenberg and Schrödinger QM versions by developing his own ingenious approach, which considered (from a unified standpoint!) transformation properties of "bra" and "ket" vectors in Hilbert space. ${ }^{39}$

\footnotetext{
${ }^{35}$ Dirac, Principles of Quantum Mechanics, 253-75.

${ }^{36}$ Ibid., 253.

${ }^{37}$ Dirac, "Quantum Theory of the Electron," 610-24.

${ }^{38}$ Paul Dirac (1977) quoted in Mehra and Rechenberg, Historical Development of Quantum Theory, 290.

${ }^{39}$ Dirac, Principles of Quantum Mechanics, 253-75.
} 
The second decisive trait was the fact that the sought-for synthesis of SRT and quantum mechanics was carried out according to the most developed model for that time - reproducing the GRT genesis. But the unification of "geometry" and "physics" turned out to be possible due to the use of a fundamentally new mathematical apparatus - tensor calculus. The latter played the role of a neutral theoretical language for Einstein, which made it possible to compare and therefore unite particular theoretical schemes of non-metric theories of Abraham, Nordström and Einstein himself. As Dirac himself later stressed in his article "Quantum Singularities in an Electromagnetic Field":

The steady progress of physics requires for its theoretical formulation a mathematics that gets continually more advanced. This is only natural and to be expected. What, however, was not expected by the scientific workers of the last century was the particular form that the line of advancement of the mathematics would take, namely, it was expected that the mathematics would get more and more complicated, but would rest on a permanent basis of axioms and definitions, while actually the modern physical developments have required a mathematics that continually shifts its foundations and gets more abstract. Non-euclidean geometry and non-commutative algebra, which were at one time considered to be purely fictions of the mind and pastimes for logical thinkers, have now been found to be very necessary for the description of the general facts of the physical world. It seems likely that this process of increasing abstraction will continue in the future and that advances in physics is to be associated with a continual modification of the axioms at the base of mathematics rather than with a logical development of any one mathematical scheme on a fixed foundation. ${ }^{40}$

Yet while Einstein generalized the available theoretical schemes by means of the language of tensor calculus, then Dirac generalized the theoretical schemes of Klein-Gordon-Fock and Pauli-Heisenberg-Jordan using no less powerful and advanced language of the theory of bispinors and Clifford algebra.

And, finally, the third hallmark of synthesis consisted in the fact that Dirac learned the specifics of the GTR synthesis not via personal contacts with its creator or careful study of primary sources, but through the peculiar prism of his Cambridge colleague and mentor - the English astronomer Sir Arthur Eddington, the author of the best (according to Einstein himself)

${ }^{40}$ Dirac, "Quantized Singularities in the Electromagnetic Field," 60 (my emphasis). 
exposition of STR and GTR - monograph Mathematical Theory of Relativity (1920). Note that for Eddington the mathematical constructs of geometry - all those tensors, vectors and "square roots of vectors" - were certain primordial bricks for laying the foundation of the Universe grounded on "synthesis of representations" in accordance with his Husserlian ideas in the spirit of the quirk theories of Cartan and Weyl. Therefore, having perceived the methods of constructing dual (hybrid) objects, containing, among other things, recipes for "interpreting new mathematical properties in terms of physical quantities (using a process similar to Eddington's Identification Principle)," Dirac was in no hurry to reveal the very philosophical principles of Eddington's philosophy. The odd principles evoked an extremely negative reaction from the majority of Copenhagen-minded physicists (and Pauli even christened them - albeit in one of his letters, not publicly! - "complete nonsense"). Although, when it came to other Eddington influences that had a significant impact on the science of the $20^{\text {th }}$ century - whether it was the staggering cosmological "hypothesis of large numbers," which led to the anthropic principle, or the adventurous $\delta$-function - Dirac did not hesitate to connect with Eddington:

I noticed the need for it [i.e., the $\delta$-function] very much in a certain place of Eddington's book on The Mathematical Theory of Relativity [in a footnote on page 190 of the 1923 edition]. ${ }^{41}$

Therefore, emphasizing the pivotal role of the "principle of mathematical beauty" in the construction of new theories, Dirac cautiously noted that the latter is inherent in all objects of Nature and therefore can be disclosed by the theoretician in his theoretical models. True, the question inevitably arises of why Nature is arranged in this way, and who nevertheless tried to arrange it in this way too. But Dirac, apparently, forwarded the question to philosophers (and theologians?), specifying that "this is a quality which cannot be defined, any more than beauty in art can be defined, but which people who study mathematics usually have no difficulty in appreciating." ${ }^{\prime 2}$

${ }^{41}$ Paul Dirac quoted in Mehra and Rechenberg, Historical Development of Quantum Theory, 81.

${ }^{42}$ Dirac, "Relation between Mathematics and Physics," 123. 


\section{Acknowledgements:}

It is a pleasure to thank the unknown reviewers for helpful critical remarks.

\section{Bibliography:}

Bjorken, David, and Sydney Drell. Relativistic Quantum Mechanics. New York: McGraw-Hill Education, 1998.

Bokulich, Alisa. "Open or Closed? Dirac, Heisenberg and the Relation between Classical and Quantum Mechanics." Studies in History and Philosophy of Science 35, no. 3 (2004): 377-96. https://doi.org/10.1016/j.shpsb.2003.11.002.

Darrigol, Olivier. From c-Numbers to q-Numbers: The Classical Analogy in the History of Quantum Theory. Berkeley: University of California Press, 1992. https://doi.org/10.1525/9780520328280.

Dirac, Paul. "The Physical Interpretation of the Quantum Dynamics." Proceedings of the Royal Society of London, A 113 (1927): 621-41.

https://doi.org/10.1098/rspa.1927.0012.

Dirac, Paul. "The Quantum Theory of the Electron." Proceedings of the Royal Society of London, A 117 (1928): 610-24. https://doi.org/10.1098/rspa.1928.0023.

Dirac, Paul. "Quantized Singularities in the Electromagnetic Field." Proceedings of Royal Society of London, A 133 (1931): 60-72.

https://doi.org/10.1098/rspa.1931.0130.

Dirac, Paul. "The Relation between Mathematics and Physics." Proceedings of the Royal Society of Edinburgh, A 59 (1938-1939): 122-29.

https://doi.org/10.1017/S0370164600012207.

Dirac, Paul. The Principles of Quantum Mechanics. Oxford: Clarendon Press, 1981.

Eddington, Arthur. Space, Time and Gravitation. An Outline of General Relativity Theory. Cambridge: Cambridge University Press, 1920.

Eddington, Arthur. The Mathematical Theory of Relativity. Cambridge: Cambridge University Press, 1920.

Heisenberg, Werner, and Pascual Jordan. "Andwendung der Quantenmechanik auf der Problem der anomalen Zeemaneffekte." Zeitschrift fur Physik 37 (1926): 263-77. https://doi.org/10.1007/BF01397100.

Heisenberg, Werner. Physik und Philosophie. Stuttgart: Hirzel, 2000. 
Kragh, Helge. "The Genesis of Dirac's Relativistic Theory of Electrons." Archive for History of Exact Sciences 24 (1981): 31-67. https://doi.org/10.1007/BF00327714.

Kragh, Helge. Dirac. A Scientific Biography. New York: Cornell University, 1990.

Mehra, Jagdish, and Helmut Rechenberg. The Historical Development of Quantum Theory. Volume 6: The Completion of Quantum Mechanics 1926-1941. New York: Springer, 2000.

Nugayev, Rinat M. "Communicative Rationality of the Maxwellian Revolution." Foundations of Science 20, no. 4 (2015): 447-78.

https://doi.org/10.1007/s10699-015-9417-z.

Nugayev, Rinat M. Einstein's Revolution: A Study in Theory Unification. Sharjah: Bentham Science Publishers, 2018. https://doi.org/10.2174/97816810863541180101.

Nugayev, Rinat M. The Planck-Einstein Breakthrough: Reconciliation of the Pivotal Research Programs of the Classical Physics. Montreal: Minkowski Institute Press, 2020.

Pauli, Wolfgang. “Über das Wasserstoffspektrum vom Standpunkt der neuen Quantenmechanik.” Zeitschrift fur Physik 36 (1926): 336-63. https://doi.org/10.1007/BF01450175.

Ryckman, Thomas. The Reign of Relativity. Philosophy in Physics 1915-1925.

Oxford: Oxford University Press, 1982.

Schrödinger, Erwin "Quantisierung als Eigenwertproblem." Annalen der Physik 384, no. 4 (1926): 361-76. https://doi.org/10.1002/andp.19263840404.

Stepin, Vyacheslav. Theoretical Knowledge. Netherlands: Springer, 2005. https://doi.org/10.1007/1-4020-3046-0.

Valente, Mario. "The Dirac Equation and its Interpretations." Preprint, submitted April 2020. https://www.researchgate.net/publication/340664785.

Weyl, Hermann. Raum, Zeit, Materie. Berlin: Springer, 1919. https://doi.org/10.1007/978-3-662-43111-5. 